\title{
RESISTÊNCIA "IN VIVO" DO PLASMODIUM FALCIPARUM ÀS 4-AMINO - QUINOLEÍNAS E À ASSOCIAÇĀO SULFADOXINA-PIRIMETAMINA. I - ESTUDO DE PORTO VELHO, RONDÔNIA, 1983.
}

\author{
Santiago Reyes, Afonso D. C. Passos e Carlos H. Osanai.
}

\begin{abstract}
Através da prova de 7 dias foi estudado o grau de resistência do Plasmodium falciparum à cloroquina, amodiaquina e sulfadoxina-pirimetamina em Porto Velho, Estado de Rondônia, Brasil. Não se observaram diferenças significativas nas médias de parasitas nos dias de seguimento e nas proporções de resistência entre os três medicamentos testados, fazendo com que os autores recomendem a manutenção das 4-aminoquinoleinas como drogas a serem usadas atualmente em infeç̧ōes não graves por P. falciparum na área de Porto Velho.
\end{abstract}

Palavras chave: Malária. Terapêutica. Resistência medicamentosa. Amazônia.

O problema da resistência do Plasmodium falciparum a drogas antimaláricas vem sendo detectado progressivamente em várias partes do mundo, acarretando dificuldades crescentes no manejo clínico de doentes e nas atividades de combate à endemia.

Entre nós a questão não é recente, uma vez que, já em 1961, foi documentada resistência "in vivo" à cloroquina em pacientes oriundos de diversas áreas da Amazônia 13. Estudos posteriores realizados "in vivo" e "in vitro" confirmaram este achadol 361012 , fazendo com que a eficácia da cloroquina na terapêutica da malária causada por $P$. falciparum venha sendo seguidamente colocada em dúvida. Uma outra 4-aminoquinoleina, a amodiaquina, tem sido postulada por alguns autores como mais ativa que a cloroquina na sua atividade esquizonticida sangüinea ${ }^{711}$, embora a resistência a essa droga se verifique hoje de maneira difusa e a sua real utilidade na luta antimalárica esteja sendo, por isso, contestada.

Como uma alternativa às 4-aminoquinoleinas, iniciou-se o emprego da associação sulfadoxinapirimetamina, obtendo-se, de imediato, excelentes resultados na cura clínica e negativação laboratorial de pacientes comprovadamente resistentes a outras drogas ${ }^{8}$. Entretanto, também a essa associação verificou-se resistência com o passar do tempo 24 , muito embora conserve ainda hoje o caráter de

Divisão de Epidemiologia da SUCAM - Esplanada dos Ministérios, Anexo do Ministério da Saúde, Ala "B" - 3o andar, sala 318. Brasília, DF.

Recebido para publicação em 6/9/84. alternativa de primeira escolha em casos de resistência às 4-aminoquinoleínas.

A Superintendència de Campanhas de Saúde Pública (SUCAM) do Ministério da Saúde, instituição que tem sob sua responsabilidade direta a execução das ações antimaláricas no Brasil, vem utilizando as 3 drogas supracitadas nas suas atividades de rotina. Dada a importância de que se reveste o conhecimento do grau de resistência a essas drogas em diferentes áreas da Amazônia para o correto direcionamento das suas ações, a SUCAM optou pelo desenvolvimento de uma investigação inicial em quatro áreas críticas de malária no Brasil: Rondônia, Maranhão, Pará e Amazonas. O presente trabalho refere-se à pesquisa realizada na cidade de Porto Velho, capital do Estado de Rondônia.

\section{MATERIAL E MÉTODOS}

Foram estudados 92 portadores de malária por $P$. falciparum selecionados da demanda normal do posto de diagnóstico e tratamento situado na Diretoria Regional da SUCAM em Porto Velho. Para ser incluido no estudo, o paciente deveria preencher os seguintes critérios de seleção:

a - apresentar infecção única por $P$. falciparum com densidade entre 1000 e 10000 formas assexuadas por $\mathrm{mm}^{3}$ de sangue, diagnosticada pela técnica da gota espessa;

b - não apresentar forma clinicamente grave;

$\mathrm{c}$ - permanecer por um período minimo de 7 dias próximo ao centro onde o estudo seria conduzido. 
Reyes S, Passos ADC, Osanai CH. Resistência "in vivo" do Plasmodium falciparum às 4-aminoquinoleinas e à associação sulfadoxina-pirimetamina. I. Estudo de Porto Velho, Rondônia, 1983. Revista da Sociedade Brasileira de Medicina Tropical 18: 175-181, Jul-Set, 1985

Os pacientes foram alocados aleatoriamente em 3 grupos, correspondendo cada um deles a um esquema de tratamento. $O$ grupo $A$ ( 31 participantes) recebeu cloroquina na dose total de $25 \mathrm{mg} / \mathrm{kg}$ de peso, distribuida em 3 dias, segundo o clássico esquema de tratamento de caso agudo ( 4 comprimidos no 1 \% dia e 3 nos dois dias seguintes, para adultos). $O$ grupo $B$ ( 31 participantes) recebeu amodiaquina em doses iguais às da cloroquina e ao grupo C ( 30 participantes) coube a associação sulfadoxina-pirimetamina (da CEME) na dosagem de 2 comprimidos no 1 . dia e um no dia seguinte, para adultos. Individuos abaixo de 15 anos, nos 3 grupos, foram medicados segundo a posologia recomendada pelo Manual de Terapêutica da Malária da SUCAM ${ }^{14}$. As drogas foram sempre ministradas sob o controle direto de uma pessoa envolvida na investigação.

Convencionando-se chamar de dia zero aquele em que o diagnóstico foi feito e a primeira dose do medicamento ministrada, os pacientes foram seguidos nos dias $1,2,3,5$ e 7 quando novas contagens de parasitas foram realizadas. Esta estratégia de acompanhamento baseou-se na prova prática padronizada de 7 dias preconizada pela Organização Mundial da Saúde (OMS) para determinação de resistência à cloroquina ${ }^{9}$. Os resultados de tal prova são assim interpretados:

- Quando não se encontram formas assexuadas de parasitas nos dias 6 e 7, os mesmos podem ser tanto sensiveis como resistentes em grau RI (tardio).

- Quando formas assexuadas de parasitas desaparecem por pelo menos 2 dias mas reaparecem $e$ se fazem presentes no dia 7, a infecção é considerada resistente em grau RI (precoce).

- Quando os parasitas assexuados não desaparecem durante o seguimento, mas têm a sua contagem reduzida a $25 \%$ ou menos de seu nivel inicial durante as primeiras 48 horas do tratamento, a infecção é considerada resistente em grau RII.

- Quando a redução da parasitemia assexuada é menor do que $75 \%$ durante as primeiras 48 horas ou a parasitemia permanece a mesma ou aumenta, a infecção é considerada resistente em grau RIII.

Por razões de ordem operacional os pacientes não foram seguidos nos dias 4 e 6 . Por esse motivo, para a classificação de Susceptivel (S) ou Resistente
Grau I (RI) levou-se em consideração a contagem

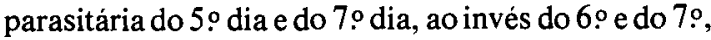
conforme classicamente estabelecido.

Os indivíduos dos grupos A e B foram submetidos à prova de detecção de 4-aminoquinoleínas na urina (método de Dill e Glazko) antes do inicio do tratamento e no dia 2, enquanto que os do grupo $\mathrm{C}$ tiveram realizada, nos mesmos dias, a prova de detecção de sulfamidas (método de Souza e Cavalcanti) na urina. Decidiu-se pela não inclusão desses resultados como um critério de seleção "a priori" dos participantes, deixando-se a consideração dos mesmos para a análise de cada caso realizada "a posteriori”".

As lâminas foram colhidas em duplicata, sendo uma examinada localmente por um microscopista experiente, mediante a contagem total das formas assexuadas em 100 campos e sua multiplicação por 5, expressando-se o resultado por milimetro cúbico. As cópias, juntamente com os dados consolidados de cada paciente, foram enviadas para revisão ao Laboratório Central da SUCAM, em Brasilia.

As perdas em cada grupo de estudo, representadas por abandono do seguimento ( 3 no grupo A, 2 no grupo $\mathrm{B}$ e 5 no grupo $\mathrm{C}$ ) e por agravamento do quadro clínico durante o tratamento ( 1 no grupo A e 1 no grupo $B$ ) foram respostas automaticamente mediante a inclusão do individuo imediatamente seguinte que comparecesse ao serviço e que satisfizesse os critérios de seleção. Os dois pacientes em que ocorreu agravamento do quadro clínico foram encaminhados para tratamento alternativo usado de rotina.

\section{RESULTADOS}

A Tabela 1 mostra a distribuição dos pacientes segundo a droga utilizada, o sexo e a idade. Verifica-se um predominio constante do sexo masculino nos 3 esquemas terapêuticos. Entre os homens o maior contingente é representado pelas faixas etárias entre 15 e 34 anos, em todos os grupos ( $70,3 \%$ no total), com uma participação proporcionalmente menor de individuos abaixo de 15 e acima de 34 anos de idade. Entre as mulheres ocorre uma distribuição mais homogênea entre as faixas etárias abaixo de $\mathbf{4 5}$ anos e apenas 1 caso acima desta idade. 
Reyes S, Passos ADC, Osanai CH. Resistência "in vivo" do Plasmodium falciparum às 4-aminoquinoleinas e à associação sulfadoxina-pirimetamina. I. Estudo de Porto Velho, Rondonia, 1983. Revista da Sociedade Brasileira de Medicina Tropical 18: 175-181, Jul-Set, 1985

A Tabela 3 apresenta os graus de resistência observadas às drogas utilizadas. Conforme a explicitação dos critérios utilizados, a categorização $S$ ou RI deve-se ao fato de não ser possivel diferenciar susceptibilidade de resistência tardia grau $\mathrm{I}$ em seguimentos de 7 dias, uma vez que recrudescências podem surgir após esta época em indivíduos laboratorialmente negativos no dia 7 . Os 3 grupos apresentam proporções semelhantes de S ou RI $(64,5 \%$ para cloroquina, $71,0 \%$ para amodiaquina e $66,7 \%$ para sulfa-pirimetamina). Deste último grupo, 93,4\% concentram-se nas categorias "S ou RI", contra $77,4 \%$ e $80,7 \%$ dos grupos de cloroquina e da amodiaquina, respectivamente. Agrupando-se os valores correspondentes a RII e RIII: $\mathrm{X}^{2} 4 \mathrm{GL}=5,7$; $p>0,25$. Comparando-se os grupos da cloroquina $\mathrm{e}$ da sulfa-pirimetamina: $\mathrm{X}^{2} \mathrm{GL}=4,0 ; \mathrm{p}>0,10$.

Tabela 3 - Grau de resistência do Plasmodium falciparum em pacientes submetidos a tratamento com cloroquina, amodiaquina e sulfa-pirimetamina. Porto Velho, Rondônia, 1983.

\begin{tabular}{|c|c|c|c|c|c|c|c|c|c|}
\hline \multirow{2}{*}{$\begin{array}{l}\quad \text { Droga } \\
\text { Grau de } \\
\text { resistência }\end{array}$} & \multicolumn{3}{|c|}{ Cloroquina } & \multicolumn{3}{|c|}{ Amodiaquina } & \multicolumn{3}{|c|}{ Sulfa-pirimetamina } \\
\hline & $N o$ & $\%$ & $\% a c . *$ & $N o$ & $\%$ & $\% a c$. & $N o$ & $\%$ & $\% a c$ \\
\hline S ou RI & 20 & 64,5 & 64,5 & 22 & 71,0 & 71,0 & 20 & 66,7 & 66,7 \\
\hline RI & 4 & 12,9 & 77,4 & 3 & 9,7 & 80,7 & 8 & 26,7 & 93,4 \\
\hline RII & 6 & 19,4 & 96,8 & 6 & 19,4 & 100,0 & 1 & 3,3 & 96,7 \\
\hline RIII & 1 & 3,2 & 100,0 & - & - & 100,0 & 1 & 3,3 & 100,0 \\
\hline Total & 31 & 100,0 & 100,0 & 31 & 100,0 & 100,0 & 30 & 100,0 & 100,0 \\
\hline
\end{tabular}

* \% ac. $=$ percentagem cumulativa.

\section{DISCUSSÃO}

Este trabalho é parte de uma investigação que tem como objetivo levantar subsídios que permitam uma visão da situação de resistência do $P$. falciparum a certas drogas em determinadas áreas criticas de transmissão malárica na Amazônia.

Não se pretende com ele estabelecer o mapeamento da resistência, mas sim fornecer respostas imediatas sobre a conveniência ou não de se prosseguir no uso das drogas de rotina da SUCAM, nas áreas estudadas.

Optou-se pela prova de 7 dias, julgada capaz de fornecer estas respostas e que, embora insuficiente para determinar a resistência real por não ser adequada para detectar recrudescências que ocorram após $\circ 7$ ㅇ dia oferece a vantagem de ser operacionalmente mais simples, economicamente mais viável e passivel de ser realizada sem alteraçōes profundas no trabalho de rotina.

O posto de atendimento de Porto Velho é procurado para diagnóstico e tratamento de malária por individuos residentes na própria cidade, áreas vizinhas e por aqueles em trânsito. E razoável aceitarse que a população de estudo seja representativa do que se passa em Porto Velho e áreas próximas, incluindo algumas regiões de garimpo.

O predomínio na amostra de indivíduos do sexo masculino, especialmente entre 15 e 34 anos, reflete, provavelmente, a participação de fatores de ordem profissional ligados ao risco de se contrair a doença.

Apesar do caráter aleatório que foi atribuido à alocação dos indivíduos nos diferentes grupos de estudo-demonstrado pela distribuição semelhante no que diz respeito às variáveis sexo e idade -, as médias de parasitas.no dia zero diferem nos seus valores, com o grupo da sulfa-pirimetamina apresentando diferença significativa para menos. Apesar disso, a redução observada na densidade parasitária nos 3 esquemas obedece a um padrão semelhante de queda e, embora a resposta à cloroquina aparente ter sido menos satisfatória que as demais, especialmente à sulfa-pirimetamina, não existem diferenças significativas nas médias em todos os dias de seguimento, com valores que tendem a se aproximar à medida que o tempo passa. 
Reyes S, Passos ADC, Osanai CH. Resistência "in vivo" do Plasmodium falciparum às 4-aminoquinoleinas e à associação sulfadoxina-pirimetamina. I. Estudo de Porto Velho, Rondonia, 1983. Revista da Sociedade Brasileira de Medicina Tropical 18: 175-181, Jul-Set, 1985

$\mathrm{Na}$ categoria $\mathrm{S}$ ou $\mathrm{RI}$, as proporções apresentadas pelos 3 esquemas são praticamente iguais, confirmando os resultados anteriores que indicaram comportamento semelhante na eficácia das 3 drogras. $O$ grupo da sulfa-pirimetamina mostra uma concentração maior de indivíduos até a categoria RI, sugerindo que essa combinação de drogas possa ser mais efetiva que as demais em termos de apenas raramente apresentar resistência de intensidade maior. Entretanto, agrupando-se as categorias RII e RIII, não se observam diferenças estatisticamente significativas, quer quando se comparam as $\mathbf{3}$ drogas em conjunto, quer quando se coteja a sulfa-pirimetamina com a cloroquina.

Os resultados desta investigação concordam, ao menos parcialmente, com grande número de publicações que têm referido presença de resistência do $P$. falciparum à cloroquina 136101213 e também à associação sulfadoxina-pirimetamina 24 na Amazônia. Com efeito, tal resistência faz-se presente em certo grau na população estudada em Porto Velho, embora chame a atenção o fato desse fenômeno aparecer em níveis similares nos 3 esquemas terapêuticos testados. Não se confirmam, para a área estudada, os dados de literatura que mostram ser a amodiaquina superior à cloroquina ${ }^{711}$ e ser a associação sulfadoxina-pirimetamina mais poderosa que estas contra $\boldsymbol{P}$. falciparum.

Torna-se necessário reconhecer que algumas particularidades deste trabalho servem como limitantes de conclusões definitivas. Como referido anteriormente, estudos de 7 dias não diferenciam susceptiveis de resistentes grau I, fazendo com que eventuais diferenças existentes no comportamento da parasitemia após o $70^{\circ}$ dia nos diversos esquemas terapêuticos não sejam passiveis de detecção. Perdese com isso a possibilidade de se chegar a conclusões definitivas sobre resistência, e, no caso deste estudo em particular, fica-se sem uma definição precisa de qual seja o significado da discreta inflexão ascendente mostrada no 5 \% para o 7. dia nos 3 grupos de estudo (Figura 1).

Figura 1 - Densidade parasitária de formas assexuadas de $\mathrm{P}$. falciparum em individuos submetidos a tratamento com cloroquina, amodiaquina e sulfadoxina-pirimetamina segundo dias de seguimento. Porto Velho, Rondônia, 1983.

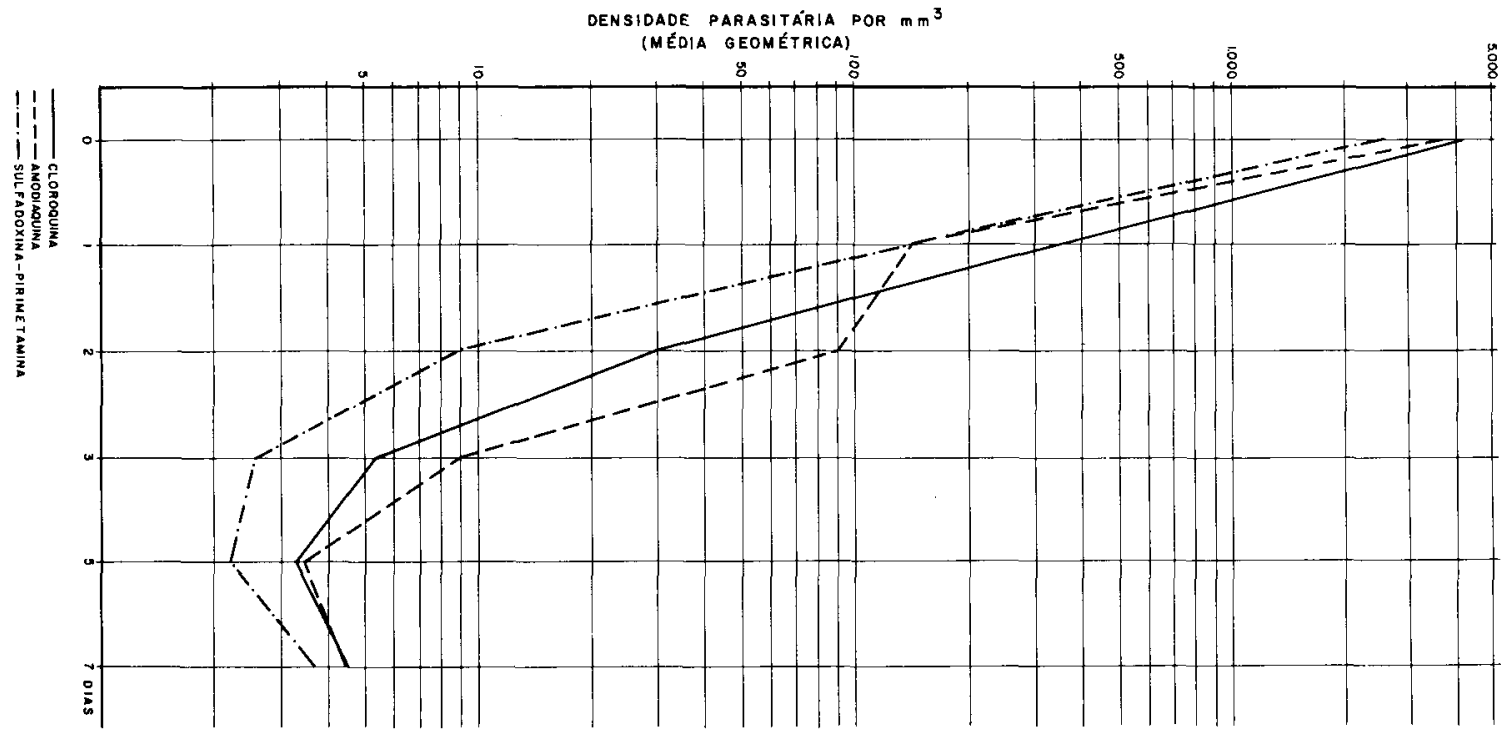

Ainda assim, considerando que esta prova permite a deteç̧ão de graus de resistência mais acentuados - RI precoce, RII e RIII -, há condições de se estimar a capacidade das drogas de prevenirem manifestações imediatas mais graves da doença, mesmo que eventuais recrudescências possam ocorrer.
Outra consideração a ser feita prende-se ao limite máximo de parasitemia adotado como critério de seleção. Por razões de ordem ética, decidiu-se limitar em 10000 o número de formas assexuadas por $\mathrm{mm}^{3}$ de sangue, não se incluindo no estudo individuos com parasitemias superiores a este valor. As conclusões da investigação devem forçosamente considerar 
Reyes $S$, Passos ADC, Osanai CH. Resistência "in vivo" do Plasmodium falciparum às 4-aminoquinoleinas e à associação sulfadoxina-pirimetamina. I. Estudo de Porto Velho, Rondônia, 1983. Revista da Sociedade Brasileira de Medicina Tropical 18: 175-181, Jul-Set, 1985

este fato, uma vez que, ao menos do ponto de vista teórico, podem existir variaçōes na resposta a drogas em função de densidades parasitárias mais elevadas.

Apesar dessas limitações, estudos deste tipo são capazes de fornecer respostas rápidas a questões que se colocam no dia-a-dia de uma instituição que trabalha em controle de malária. $O$ seu caráter de pesquisa operacional, aliando baixo custo com operacionalização simples, recomenda a sua realização periódica nas áreas onde a resistência a drogas se coloca como um obstáculo às atividades de controle. Essa periodicidade permitirá uma monitorização efetiva da área, orientando para eventuais correções de conduta que se fizerem necessárias.

As 4-aminoquinoleinas são de menor custo e apresentam menos efeitos tóxicos potenciais que a combinação sulfadoxina-pirimetamina, tendo mostrado, no estudo, eficácia semelhante a esta combinação no tratamento de casos não graves de infecções por P. falciparum. Assim, com base nos resultados desta investigação, e considerando-se que na luta antimalárica uma droga pode ser bastante útil mesmo quando a ela se verifica certo grau de resistência ${ }^{5}$, não se colocam, no momento, restriçōes ao uso das 4-aminoquinoleinas como drogas de primeira linha no tratamento de infecçōes dessa natureza na área de Porto Velho.

\section{SUMMARY}

The authors studied the level of resistance of Plasmodium falciparum malaria to chloroquine, amodiaquine and the combination of sulfadoxine and pyrimethamine in the City of Porto Velho, State Capital of Rondonia, Brazil. Using a seven day observation test they did not find significant differences in the parasite count averages in the follow-up as well as in the proportions of resistance to the three drugs. Based upon these findings they recommend the maintenance of the 4-aminoquinolines in treating uncomplicated forms of falciparum malaria in the area of Porto Velho.

Key words: Malaria. Chemotherapy. Resistence to drugs. Amazon basin.

\section{AGRADECIMENTOS}

Os autores agradecem ao Dr. Agostinho Limeira e Maria Oliveira de Souza, respectivamente,
Diretor Regional e microscopista da SUCAM em Rondônia; a Maria Alice Fernandes Santos, microscopista do Laboratório Central em Brasilia e aos doutores Edinaldo Alves Pinheiro e Pedro Luiz Tauil, respectivamente, Diretores da Divisão de Malária e do Departamento de Erradicação e Controle de Endemias, pela colaboração na execução deste trabalho.

\section{REFERÊNCIAS BIBLIOGRÁFICAS}

1. Alecrim MGC. Estudo da resistência do Plasmodium falciparum às drogas antimaláricas "in vivo" e "in vitro" na Amazônia. Tese de mestrado, Universidade de Brasilia, Brasilia, 1981.

2. Alecrim WD, Alecrim MGC, Dourado HC, Passos LF, Wanssa E. Resistência do P. falciparum à associação sulfadoxina mais pirimetamina no Amazonas a nivel de RIII. Trabalho apresentado no XVIII Congresso da Sociedade Brasileira de Medicina Tropical, Caldas Novas, 1981.

3. Almeida Neto JC. Malária por $P$. falciparum. Tese de doutoramento, Universidade Federal de Goiás, Goiânia, 1970.

4. Almeida Neto JC, Oliveira GSC, Sampaio JAA. Resistência do $P$. falciparum à associação sulfamídicos antifolínicos na região centro-oeste do Brasil. Dados referentes ao estudo de 104 pacientes. Revista de Patologia Tropical. Goiás 3:385-393, 1972.

5. Campbell CC, Payne D, Schwartz IK, Khatib OJ. Evaluation of amodiaquine treatment of chloroquine resistant Plasmodium falciparum malaria in Zanzibar, 1982. American Journal of Tropical Medicine and Hygiene 32: 1216-1220, 1983.

6. Ferraroni JJ, Speer CA, Hayes J, Suzuki M. Prevalence of chloroquine-resistant falciparum malaria in the Brazilian Amazon. American Journal of Tropical Medicine and Hygiene 30: 526-530, 1981.

7. Hall AP, Segal HE, Pearlman EJ, Phintoyothin P, Kosakal S. Amodiaquine resistant falciparum malaria in Thailand. American Journal of Tropical Medicine and Hygiene 24:575-580, 1975 .

8. Jaroonvesama N, Harinasuta T, Muangmanee L. Recrudescence on poor response or resistance to quinine of falciparum malaria in Thailand. Journal of Tropical Medicine and Public Health 5:504-509, 1974.

9. Organização Mundial da Saúde. Quimioterapia del paludismo y resistencia a los medicamentos antipalúdicos, 132p. (Série de Informes Técnicos, 529). Genebra, 1973. 
Reyes S, Passos ADC. Osanai CH. Resisténcia "in vivo" do Plasmodium falciparum às 4-aminoquinoleinas e a associaçäo sulfadoxina-pirimetamina. I. Estudo de Porto Velho, Rondonia, 1983. Revista da Sociedade Brasileira de Medicina Tropical 18: 175-181, Jul-Set, 1985

10. Reyes S. Infecçòes maláricas por Plasmodium falciparum resistentes ao tratamento com cloroquina: situaçào ro Brasil (1960-1981). Revista Brasileira de Malariologia e Doenças Tropicais 33:109-130, 1981.

11. Rieckmann KH. Determination of the drug sensitivity of Plasmodium falciparum. Journal of the American Medical Association 217:573-578, 1971

12. Rieckmann KH, Lopez - Antuñano FJ. Chloroquine resistance of $P$. falciparum in Brazil detected by a simple in vitro method. Bulletin of the World Health Organization 45: 157-167, 1971 .

13. Silva JR, Lopes PFA, Ferreira LF, Morteo R, Naveira JV. Resistência do $P$. falciparum à ação da cloroquina. $\mathrm{O}$ Hospital 60:581-594, 1961.

14. Superintendència de Campanhas de Saúde Pública. Manual de terapéutica da malária. 82 p., Brasilia, 1982. 